\title{
Ultrafast Formation of Free-Standing 2D Carbon Nanotube Thin Films through Capillary Force Driving Compression on an Air/Water Interface
}

Peng Xiao, ${ }^{\dagger}$ Jincui Gu ${ }^{\dagger}$ Changjin Wan, ${ }^{\dagger}$ Shuai Wang, ${ }^{\dagger}$ Jiang He ${ }^{\dagger}$ Jiawei Zhang, ${ }^{\dagger *}$ Youju Huang, ${ }^{\dagger}$ Shiao-Wei Kuo, ${ }^{\ddagger}$ Tao Chen ${ }^{\dagger *}$

${ }^{\dagger}$ Ningbo Institute of Material Technology and Engineering, Key Laboratory of Graphene Technologies and Applications of Zhejiang Province, Chinese Academy of Science, Zhongguan West Road 1219, 315201, Ningbo, Zhejiang, China

${ }^{\sharp}$ Department of Material and Optoelectronic Science, National Sun Yat-Sen University, 804, Kaohsiung, Taiwan 


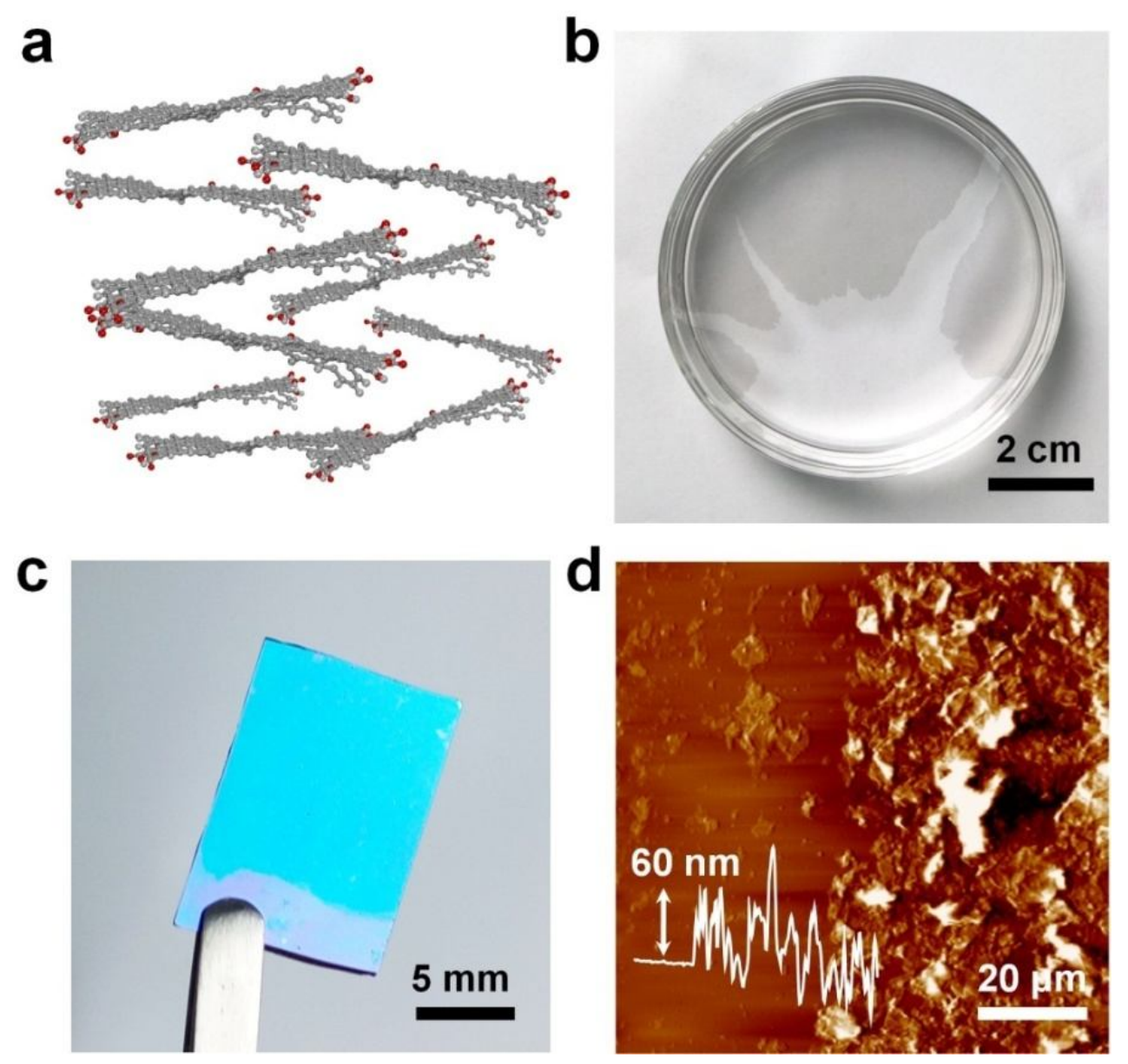

Figure S1 a) Schematic illustration of reduced graphene oxide (RGO) sheets closely packed into an integral film through $\pi-\pi$ stacking interaction. b) Photograph of RGO film. Inset: ethanol-assisted RGO dispersion. c) Photo of RGO film transferred onto the $\mathrm{SiO}_{2}$ substrate. d) AFM cross-sectional image of the RGO film, showing an ultrathin thickness of about $60 \mathrm{~nm}$. 


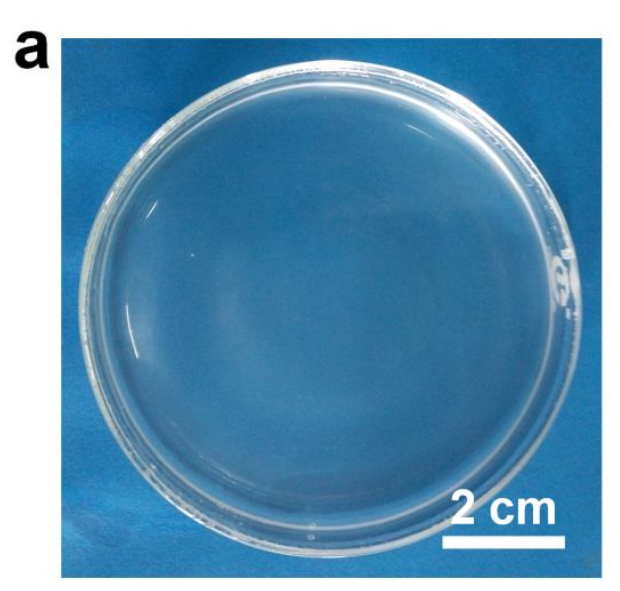

C

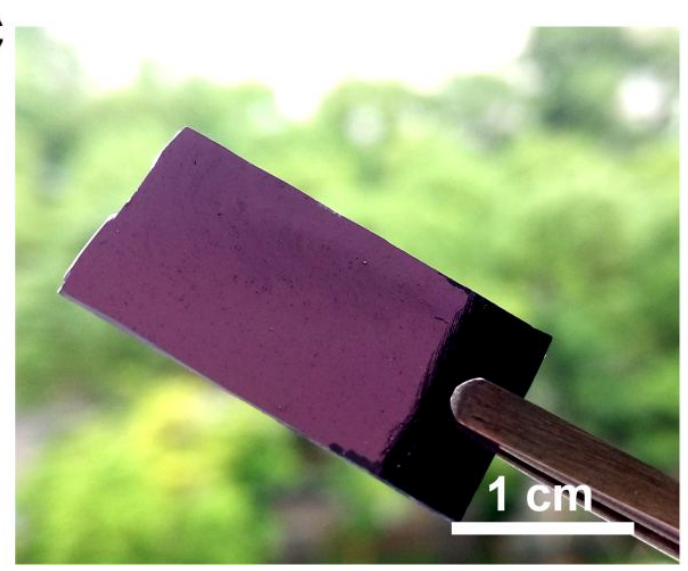

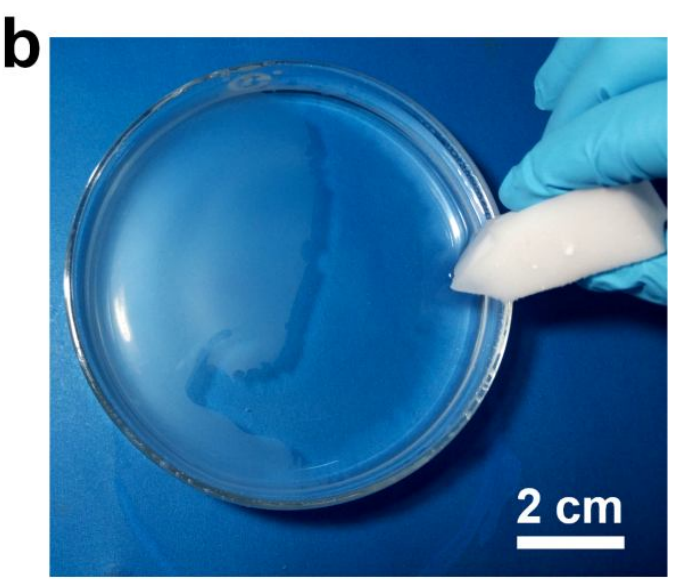
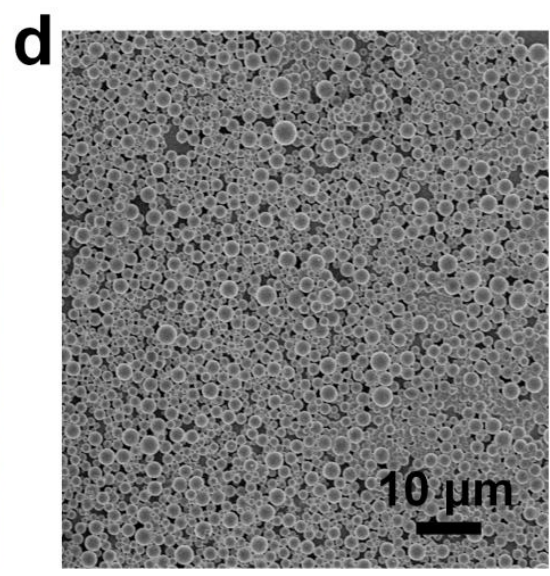

Figure S2 a) Photograph of the as-assembled $\mathrm{SiO}_{2}$ particles in the air/water interface.

b) Photograph of the successful $\mathrm{SiO}_{2}$ particles compression for compacted layers induced by the sponges driven capillary force. c) Photo of the transferred $\mathrm{SiO}_{2}$ films on $\mathrm{SiO}_{2}$ substrate. d) $\mathrm{SEM}$ image of the resulted $\mathrm{SiO}_{2}$ film, showing a highly condensed structure. 

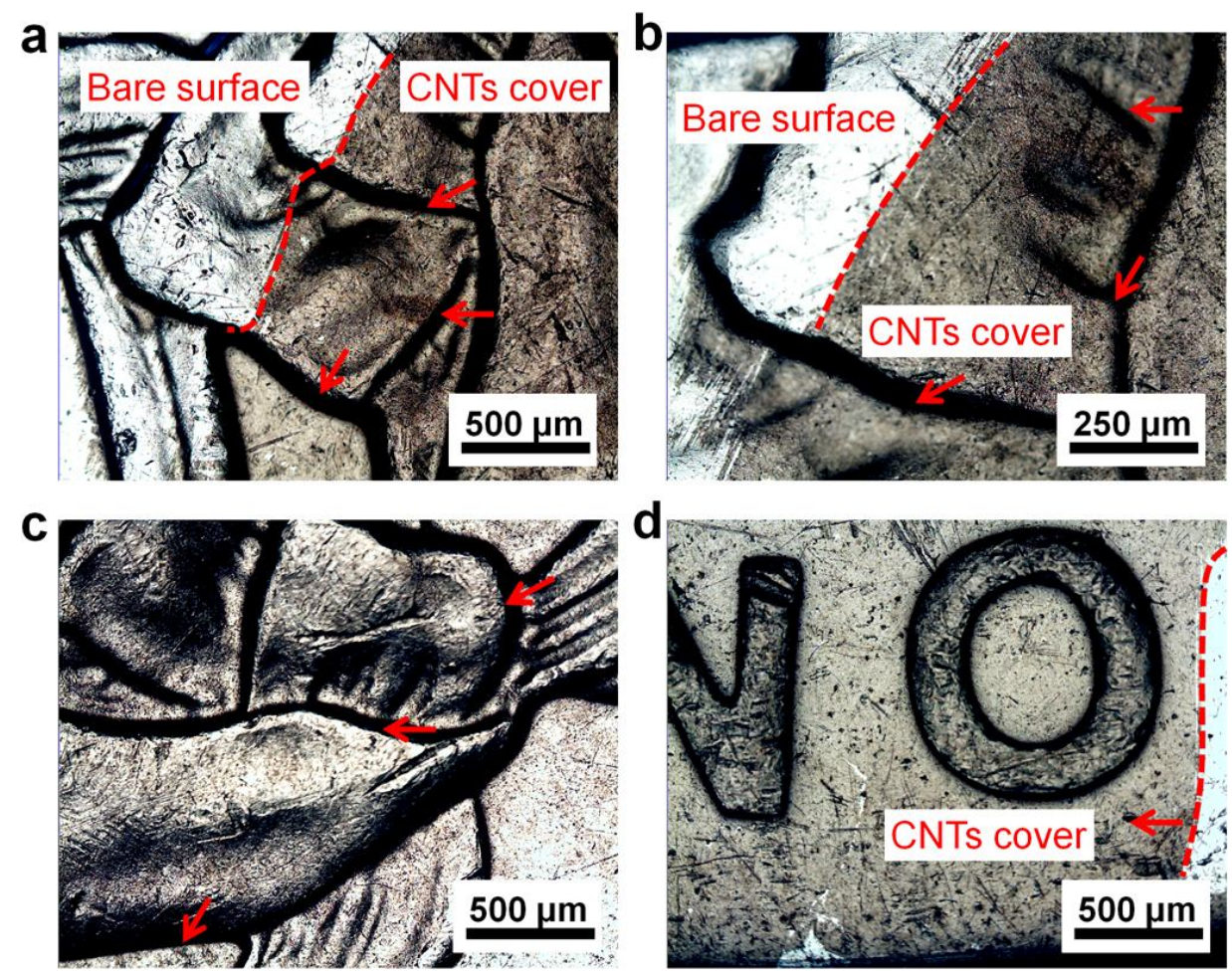

Figure S3 Optical images of the CNTs ultrathin film transferred onto the embossed metal surface a-d), showing a continuous spreading on the fluctuant surface without any breakage.

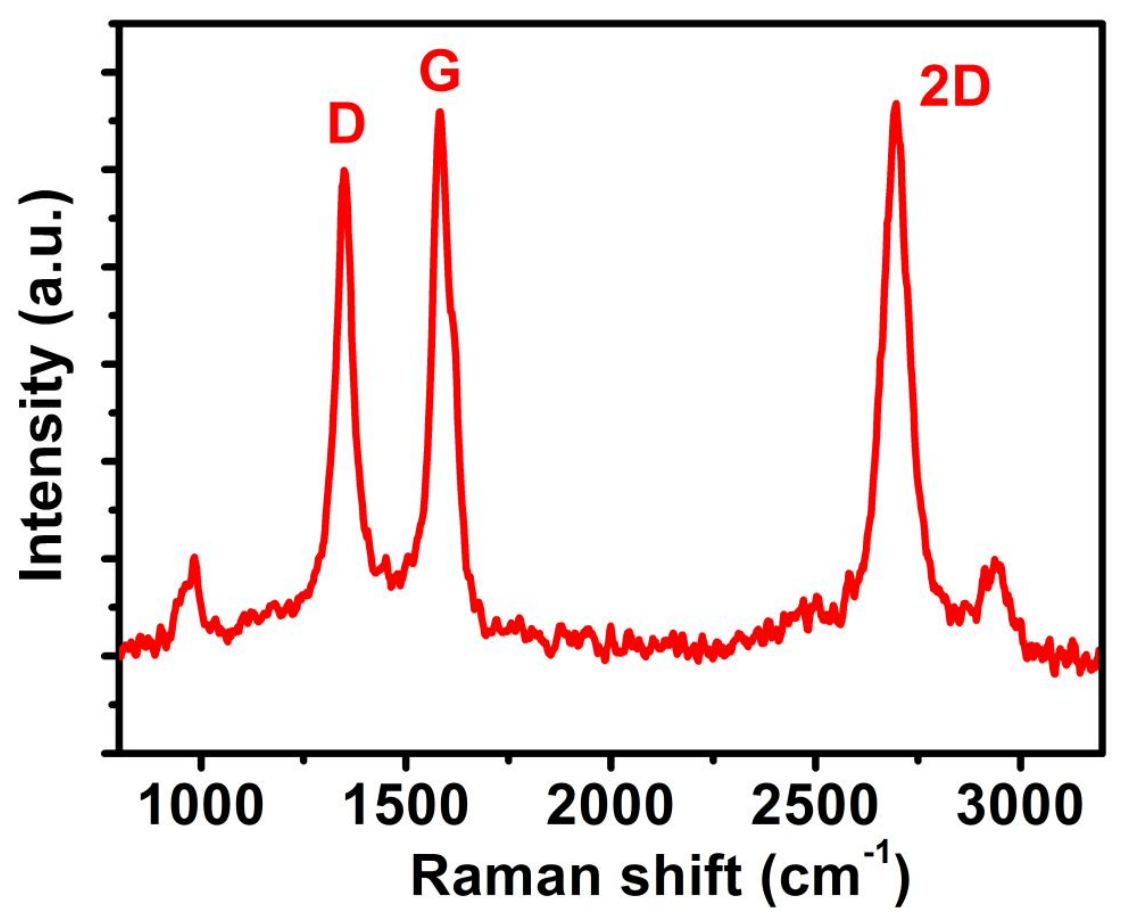

Figure S4 Raman spectrum of the CNTs. 

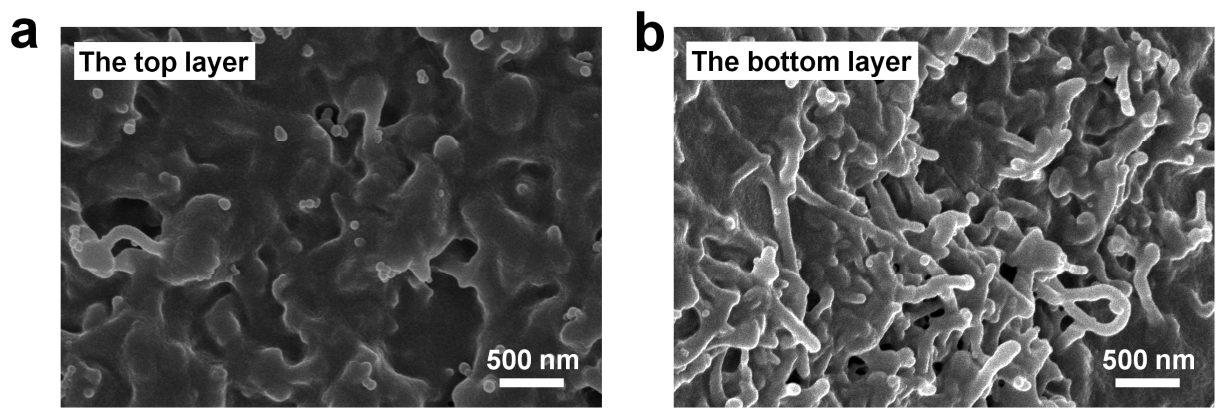

Figure S5 SEM images of the polymer grafted CNTs hybrid. a) The top layer. b) The bottom layer.
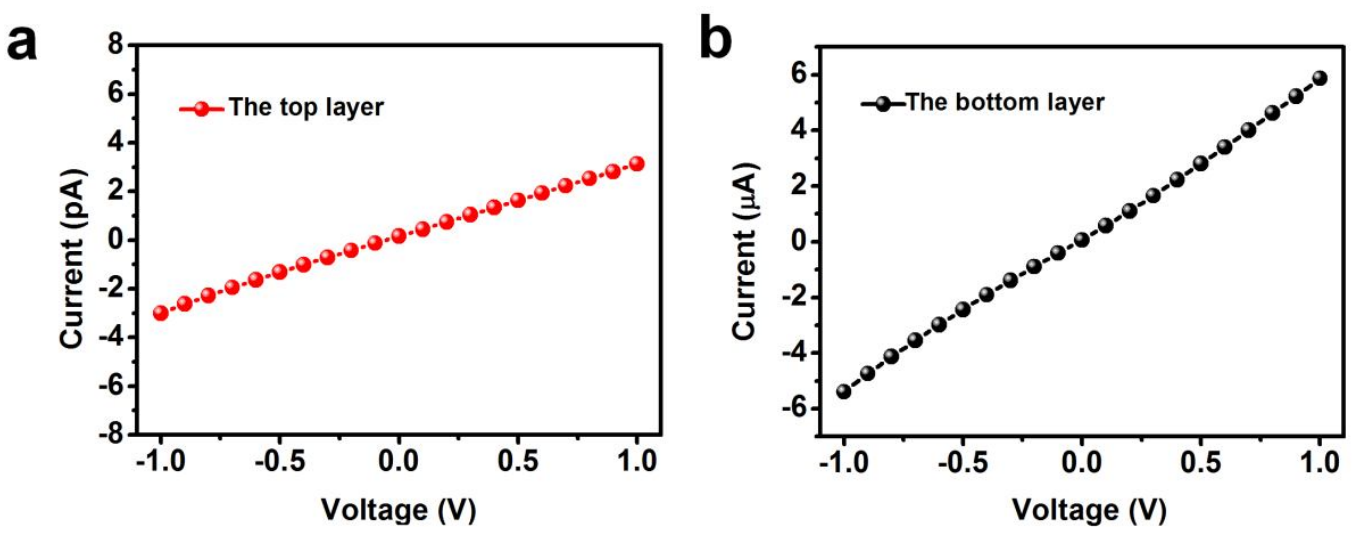

Figure S6 Current vs. voltage curves of the resulted Janus hybrid. a) The top layer. b)

The bottom layer. 